\title{
Comparison of the Effectiveness and Safety of Different Methods of Colorectal Endoscopic Submucosal Dissection: A Systematic Review and Network Meta-Analysis
}

\author{
Xueping $\mathrm{Wu}^{\mathrm{a}}$ Chenglong $\mathrm{Ye}^{\mathrm{a}}$ Zhongsheng $\mathrm{Cao}^{\mathrm{b}}$ Xiangcheng $\mathrm{Hu}^{\mathrm{b}}$ \\ Wensheng Pan ${ }^{\mathrm{a}, \mathrm{b}}$ Jiyong Jing ${ }^{\mathrm{c}}$ \\ aZhejiang Chinese Medical University, Hangzhou, China; ${ }^{b}$ Department of Gastroenterology, Zhejiang Provincial \\ People's Hospital, People's Hospital of Hangzhou Medical College, Hangzhou, China; 'Zhejiang Provincial People's \\ Hospital, People's Hospital of Hangzhou Medical College, Hangzhou, China
}

\section{Keywords}

Endoscopic submucosal dissection · Colorectal neoplasms . Network meta-analysis

\begin{abstract}
Background: Traditional endoscopic submucosal dissection (ESD) has developed different methods, such as pocket method (P-ESD), traction-assisted method (T-ESD), and hybrid method (H-ESD). In this meta-analysis, the benefits and drawbacks of different ESD methods were discussed and ranked. Study Design: Studies comparing different methods of colorectal ESD were searched by using PubMed, EMBASE, and Cochrane Library databases. The study was conducted for five endpoints: en bloc resection rate, $\mathrm{RO}$ resection rate, operation time, dissection speed, and adverse events rate. Pairwise and network meta-analyses were performed through Rev Man 5.4 and Stata 16.0. The quality of all included studies was assessed using the Cochrane Risk of Bias Tool and the Newcastle-Ottawa Scale. Results: Twenty-six studies met the inclusion criteria, including 7 RCTs and 19 non-RCTs, with a total of 3,002 patients. The pooled analysis showed that the en bloc resection rate of $\mathrm{H}$-ESD was significantly lower than that of C-ESD, P-ESD, and T-ESD (RR $=0.28,95 \%$ $\mathrm{Cl}[0.12,0.65] ; \mathrm{RR}=0.11,95 \% \mathrm{Cl}[0.03,0.44] ; \mathrm{RR}=8.28,95 \%$
\end{abstract}

$\mathrm{Cl}[2.50,27.42])$. Compared with C-ESD, the operation time of H-ESD and T-ESD was significantly shorter (MD $=-21.83$, $95 \% \mathrm{Cl}[-34.76,-8.90] ; \mathrm{MD}=-23.8,95 \% \mathrm{Cl}[-32.55,-15.06])$. Meanwhile, the operation time of T-ESD was also significantly shorter than that of P-ESD (MD $=-18.74,95 \% \mathrm{Cl}[-31.93$, $-5.54]$ ). The dissection speed of T-ESD was significantly faster than that of C-ESD (MD $=6.26,95 \% \mathrm{Cl}[2.29,10.23])$. Conclusion: P-ESD and T-ESD are probably the two best methods of colorectal ESD at present. The advantages of P-ESD are high en bloc resection rate and low incidence of adverse events. The advantages of T-ESD are rapid dissection and short operation time.

(c) 2021 S. Karger AG, Basel

\section{Introduction}

Colorectal cancer is the third most commonly diagnosed cancer and is the second leading cause of cancer death, with an estimated 1.9 million new cases (10.0\%) and an estimated 0.94 million deaths (9.4\%) in 2020 [1]. In the past decade, endoscopic submucosal dissection (ESD) has been widely used in the resection of superfi-

Xueping Wu and Chenglong Ye contributed equally. karger@karger.com

www.karger.com/ddi

(C) 2021 S. Karger AG, Base

Karger"
Correspondence to:

Wensheng Pan,wspan223@163.com

Jiyong Jing, jiyong_jing@ 126.com 
cial colorectal tumors worldwide [2]. But compared with gastric and esophageal ESD, colorectal ESD is generally considered to be more difficult to operate and has a relatively high incidence of intraoperative and postoperative complications $[3,4]$. In order to overcome these difficulties, various ESD procedures have been developed.

Traction-assisted endoscopic submucosal dissection (T-ESD), as an improvement strategy of traditional standard ESD surgery, increases submucosal visualization and maintains good maneuverability by adding a traction device during the operation [5]. Saito et al. [6] first used the traction device of the sinker system for endoscopic treatment of colorectal tumors in 2005. Subsequently, a variety of traction devices have been gradually developed, mainly including floss traction, clip-line method, clip-rubber band method, S-O clip method, suture pulley method, and other internal traction methods, as well as magnetic traction, large double balloon device, external traction method of robot platform, etc. [7-13].

Pocket-creation method (PCM) is a new ESD strategy first reported by Hayashi et al. [14]. PCM ESD (P-ESD) makes a small incision near the lesion with a needle-type knife and forms a wide pocket under the lesion, which can better stabilize the tip of the endoscope and provide good traction $[15,16]$.

Hybrid ESD (H-ESD) is the combination of conventional ESD (C-ESD) with snaring technique, means that after a certain amount of routine circumferential incision and submucosal dissection, the remaining lesions are removed by snare resection [17]. The biggest advantage of $\mathrm{H}$-ESD is that it can simplify the procedure and shorten the operation time [18].

According to previous literature reports, the efficacy or safety of P-ESD, H-ESD, and T-ESD in the treatment of colorectal tumors has been improved to different degrees compared with C-ESD. But to date, the comparison between P-ESD, H-ESD, and T-ESD has not been fully reported. The aim of this network meta-analysis is to further investigate the comparative effectiveness and safety of C-ESD, P-ESD, H-ESD, and T-ESD in endoscopic resection of colorectal tumors.

\section{Materials and Methods}

A systematic review was performed according to the guidelines from the preferred reporting items for systematic reviews and meta-analyses checklist (PRISMA-NMA) [19]. Institutional review board approval was not required for this type of study.

Effectiveness and Safety of Different

Methods of Colorectal ESD

\section{Search Strategy}

We systematically searched PubMed, Excerpta Medica Database (EMBASE), and the Cochrane Library databases. The last search was on April 3, 2021. Simultaneously, references of included literature studies were manually retrieved, and those with incomplete data or missing information were obtained by contacting corresponding authors by telephone or email. The search strategy combined mesh terms and free-text regarding "endoscopic submucosal dissection," "Colorectal Neoplasms," "Colonic Neoplasms," and "Rectal Neoplasms." Details of our search strategy are provided in online supplementary information material (see www. karger.com/doi/10.1159/000521377 for all online suppl. material).

\section{Inclusion/Exclusion Criteria}

We developed detailed inclusion criteria according to the PICOS guidelines: (1) patients with colorectal tumors meeting ESD indications; (2) interventions and control groups with different ESD surgical strategies, including C-ESD, T-ESD, H-ESD, or PESD; (3) outcomes include the effectiveness and safety of the procedure; (4) study types include randomized controlled studies or retrospective studies published in English. We excluded (1) studies that did not specify which type of surgery was performed in the intervention group or control group; (2) studies whose outcomes were missing or data were incomplete; (3) animal research studies, case reports, reviews, editorials, comments, letters, or studies with unavailable full text.

\section{Primary Outcomes}

(1) En bloc resection was defined as the resection of the whole tumor in one piece with the macroscopic tumor-free margins. (2) $\mathrm{R} 0$ resection was defined as histologically confirmed tumor-free lateral and vertical margins. (3) Adverse events were defined as perforation and bleeding during or within 2 weeks after surgery.

\section{Secondary Outcomes}

(1) Operation time was measured from the time of initial local injection until removal of the tumor. (2) Dissection speed was calculated by dividing the area of the resected specimen by the procedure time.

\section{Study Selection and Data Extraction}

The two authors (X.W. and Z.C.) independently screened all searched titles and abstracts to determine their eligibility and relevance for this review study. EndNote X9 software (Clarivate Analytics) was used to manage all references and remove duplicates. Further, the full text of the articles was read after preliminary screening, excluding the nonconforming studies according to the inclusion criteria and recording the reasons for exclusion. Any points of disagreement were fully discussed and any remaining conflicts resolved through a third party (C.Y.). The study selection process is summarized as a PRISMA-P flowchart, as shown in Figure 1.

Two authors (X.W. and Z.C.) used standardized tables to independently extract relevant information from each eligible study, including author, region, year, study type, patient $(n)$, age, sex ratio, tumor size, and tumor location. Outcomes included the R0 resection rate, en bloc resection rate, operation time, dissection speed, and adverse events (intraoperative or postoperative) rate. In addition, the corresponding author was contacted via email requesting sufficient original data to ensure the accuracy of this analysis. 


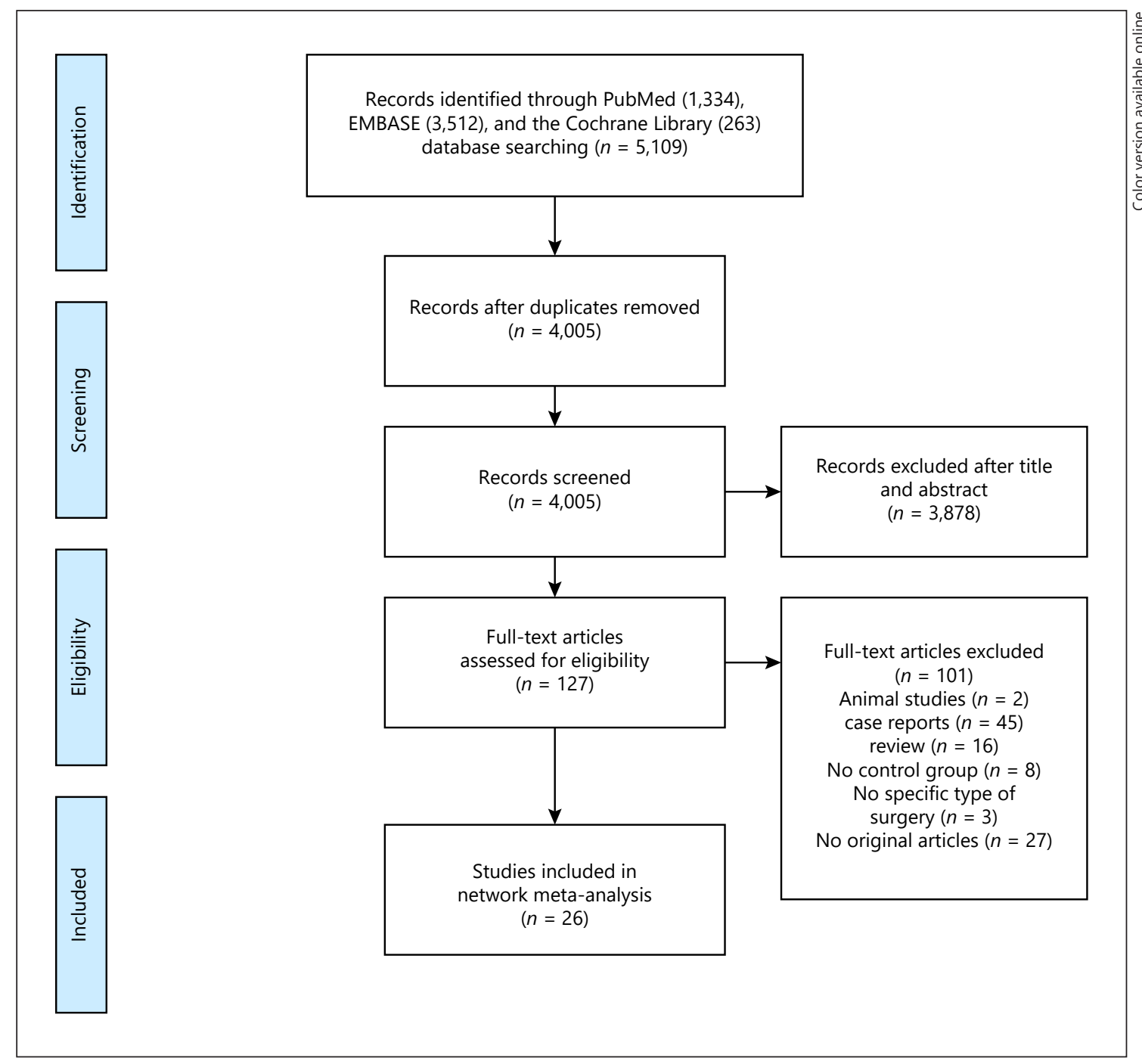

Fig. 1. Flowchart summarizing study selection process.

\section{Methodological Quality Assessment}

We evaluated the quality of RCT studies using the Cochrane Risk of Bias Tool, including random sequence generation, allocation concealment, blinding of participants and personnel, blinding of outcome assessment, incomplete outcome data, selective reporting, and other biases [20]. Each RCT study was classified as low, moderate, or high risk of bias.

Meanwhile, the Newcastle-Ottawa Scale (NOS) was used to evaluate the quality of cohort studies [21]. This is a star scoring system, which is mainly divided into three parts, namely, selection, comparability, and outcome, with a total score of 9 , and a score above 7 is considered a high-quality study. The quality evaluations of all the above included studies were independently evaluated by two independent reviewers (C.L.Y. and Z.S.C.), and the differences were discussed by a third reviewer (X.C.H.) to reach a consensus.

\section{Statistical Analysis}

Review Manager (Rev Man) Software (version 5.4; The Nordic Cochrane Centre, Copenhagen, Denmark) was used to summarize and analyze direct pairwise comparative studies, including P-ESD versus C-ESD, H-ESD versus C-ESD, T-ESD versus C-ESD, and T-ESD versus P-ESD. The network meta-analysis (NMA) was performed using the STATA 16.0 software (Stata Corporation, College Station, TX, USA), which contains direct and indirect comparisons based on the frequency school of Rücker [22]. RR (risk ratio) and $95 \%$ confidence intervals (CIs) were used to calculate the dichotomous outcomes of en bloc resection rate, $\mathrm{R} 0$ resection rate, and adverse events rate. For the continuous outcomes, such as operation time and dissection speed, the weighted mean difference (MD) was calculated. According to the methodology of Hozo et al. [23], the data reported as the median (range) were converted into the mean \pm SD. $p$ value less than 0.05 was considered statistically significant. We examined statistical heterogeneity among 
studies using the $I^{2}$ statistic and Cochrane's $\chi^{2}$ test; $I^{2} \leq 50 \%$ and $p>0.1$ were considered to have no significant heterogeneity. If there was no obvious heterogeneity in the data, the fixed effects model was used, otherwise the random effects model was adopted. Sensitivity analysis, subgroup analysis, or descriptive analysis was performed when there was significant heterogeneity. The effectiveness and safety of various ESD types were compared by the SUCRA, and they were ranked according to outcome measures. For the en bloc resection rate, $\mathrm{R} 0$ resection rate, and dissection speed, the higher the ranking, the higher the effectiveness. However, for the operation time and the adverse events rate, the lower the ranking, the more obvious the advantage. Funnel plots were used to evaluate publication bias. Symmetrical plots indicated that publication bias had little influence, while asymmetrical plots indicated that publication bias might exist.

\section{Results}

\section{Included Literature and Study Characteristics}

A total of 5,109 studies were found by searching PubMed, EMBASE, and the Cochrane Library databases. After deleting the duplicates, preliminary screening of titles and abstracts, 127 studies were included in full-text review. Finally, after screening in strict accordance with inclusion and exclusion criteria, 26 records $[15,16,24-$ 47] were included in this network meta-analysis, of which 7 were RCT studies [24-30] and 19 were retrospective cohort studies [15, 16, 31-47] (Fig. 1).

Among the 26 included studies, 6 were compared between P-ESD and C-ESD [15, 16, 24, 29, 34, 42], 5 were compared between H-ESD and C-ESD [30, 31, 39, 40, 47], 14 were compared between T-ESD and C-ESD [25-28, $32,33,35-38,41,44-46]$, and 1 was compared between T-ESD and P-ESD [43]. The study by Indo et al. [44] described two traction methods compared with the traditional method, and since there was no significant heterogeneity between the two groups of traction methods, we combined them. The study by Ide et al. [46] made a threearm comparison between the conventional method, the traction method, and the PCM with traction. We only extracted data for the comparison between the traditional method and the traction method.

The characteristics of all enrolled patients were summarized and analyzed. A total of 3,002 patients were included in this meta-analysis, 1,593 (53.1\%) of whom completed C-ESD, 610 (20.3\%) of whom completed P-ESD, 192 (6.4\%) of whom completed H-ESD, and 607 (20.2\%) of whom completed T-ESD. The baseline characteristics such as gender, age, and tumor diameter of patients undergoing C-ESD, P-ESD, H-ESD, and T-ESD are provided in Table 1.

Effectiveness and Safety of Different Methods of Colorectal ESD

\section{Quality Assessment}

Of the 19 retrospective cohort studies, 9 had a total score of 7 or greater according to the Newcastle-Ottawa Scale (NOS) quality assessment, while the remaining 10 had a total score of 6 . Since this analysis focused on shortterm complications of ESD surgery, all included studies would have scored 7 points or higher if follow-up was limited to the postoperative hospital stay, which would have been considered high-quality studies according to the NOS quality assessment criteria. Three RCTs were identified as having a high risk of performance bias because the endoscopists were not blind to the ESD procedures performed according to the Cochrane Risk of Bias Tool [26-28]. Only one study did not report the generation of random sequences; 5 studies did not report allocation hiding, and the other two reported specific allocation concealment. Moreover, 3 studies indicated blindness to the results, and other studies did not mention their outcome blinding. Most studies had no reporting bias, attrition bias, or other biases. Overall, the assessment of the quality of all the included studies was positive. The quality assessment details of the included literature are shown in Table 2 and Figure 2.

\section{Pairwise Meta-Analysis}

\section{En Bloc Resection Rate}

Twenty-three studies described direct comparisons of the en bloc resection rate. Six articles reported the en bloc resection rate compared between P-ESD and C-ESD, and the pooled analysis showed that $\mathrm{P}$-ESD had higher en bloc resection rate than C-ESD $(\mathrm{RR}=1.04,95 \% \mathrm{CI}[1.00,1.07]$, $p<0.05)$ with a very low heterogeneity $\left(I^{2}=0 \%, p=0.95\right)$. Four studies compared H-ESD with C-ESD, and analysis showed that there was no significant difference in the en bloc resection rate between $\mathrm{H}$-ESD and C-ESD $(\mathrm{RR}=0.84$, $95 \% \mathrm{CI}[0.68,1.04], p=0.12)$ with substantial heterogeneity $\left(I^{2}=80 \%, p=0.002\right)$. We performed sensitivity analysis to change the effect measure, and the results showed that $\mathrm{H}$-ESD had lower en bloc resection rate than C-ESD (OR $=0.28,95 \% \mathrm{CI}[0.17,0.47], p<0.05)$ with a relatively low heterogeneity $\left(I^{2}=43 \%, p=0.15\right)$ (online suppl. Fig. 6). Twelve articles reported the en bloc resection rate compared between T-ESD and C-ESD, and the pooled analysis showed that T-ESD was similar to C-ESD (RR $=1.02,95 \%$ CI $[0.99,1.04], p=0.18$ ) with a very low heterogeneity $\left(I^{2}=0 \%, p=0.96\right)$. One article reported the en bloc resection rate compared between T-ESD and P-ESD, and the analysis showed that T-ESD had higher en bloc resection rate than $\mathrm{P}-\mathrm{ESD}(\mathrm{RR}=1.31,95 \% \mathrm{CI}[1.16,1.49], p<0.0001)$. The results are shown in online supplementary Figure 1. 
Table 1. Baseline characteristics of all included patients

\begin{tabular}{lllll}
\hline & C-ESD & P-ESD & H-ESD & T-ESD \\
\hline $\begin{array}{llll}\text { Patients, } N \\
\text { Gender, } n(\%)\end{array}$ & 1,593 & 610 & 192 & 607 \\
$\quad$ Male & $991(62.2)$ & $377(61.8)$ & $113(58.9)$ & $345(56.8)$ \\
$\quad$ Female & $602(37.8)$ & $233(38.2)$ & $79(41.1)$ & $262(43.2)$ \\
Age (mean \pm SD), years & $66 \pm 9.9$ & $68.1 \pm 9.9$ & $65.4 \pm 11.0$ & $68 \pm 9.1$ \\
Size (mean \pm SD) & $32.1 \pm 14.3$ & $33.7 \pm 14.1$ & $26.2 \pm 10.9$ & $34.8 \pm 17.9$ \\
\hline
\end{tabular}

C-ESD, conventional endoscopic submucosal dissection; T-ESD, traction-assisted endoscopic submucosal dissection; P-ESD, pocket-creation method endoscopic submucosal dissection; H-ESD, hybrid endoscopic submucosal dissection.

Table 2. The quality of the observational studies was performed using the Newcastle-Ottawa Scale

\begin{tabular}{|c|c|c|c|c|c|c|c|c|c|}
\hline \multirow[t]{2}{*}{ Studies } & \multicolumn{4}{|c|}{ Selection } & \multirow[t]{2}{*}{ Comparability } & \multicolumn{3}{|c|}{ Outcome } & \multirow{2}{*}{$\begin{array}{l}\text { Total score } \\
\text { (out of 9) }\end{array}$} \\
\hline & ROS & SOC & $\mathrm{AOE}$ & DOI & & AOU & DOF & AFU & \\
\hline Yamashina [34] & $*$ & $*$ & $*$ & $*$ & $* *$ & $*$ & & & 7 \\
\hline Takezawa et al. [16] & $*$ & $*$ & $*$ & $*$ & $*$ & $*$ & & & 6 \\
\hline Sakamoto et al. [11] & * & $*$ & $*$ & $*$ & $* *$ & $*$ & & & 7 \\
\hline Kanamori et al. [42] & $*$ & $*$ & $*$ & $*$ & $*$ & $*$ & & & 6 \\
\hline Okamoto et al. [39] & $*$ & $*$ & $*$ & $*$ & $*$ & $*$ & & & 6 \\
\hline Milano et al. [40] & $*$ & $*$ & $*$ & $*$ & $*$ & * & $*$ & $*$ & 8 \\
\hline Terasaki [31] & $*$ & $*$ & $*$ & $*$ & $*$ & $*$ & $*$ & & 7 \\
\hline Byeon et al. [47] & $*$ & $*$ & $*$ & $*$ & $*$ & $*$ & $*$ & $*$ & 8 \\
\hline Ye et al. [32] & $*$ & $*$ & $*$ & $*$ & $* *$ & $*$ & & & 7 \\
\hline Yamashina et al. [33] & $*$ & $*$ & $*$ & $*$ & $* *$ & $*$ & & & 7 \\
\hline Yamada et al. [35] & $*$ & $*$ & $*$ & $*$ & $*$ & $*$ & & & 6 \\
\hline Takashiro et al. [36] & $*$ & $*$ & $*$ & $*$ & $*$ & $*$ & & & 6 \\
\hline Okamoto et al. [37] & $*$ & $*$ & $*$ & $*$ & $* *$ & $*$ & & & 7 \\
\hline Okamoto et al. [38] & $*$ & $*$ & * & $*$ & $*$ & $*$ & & & 6 \\
\hline Kuroki et al. [41] & $*$ & $*$ & $*$ & $*$ & $*$ & $*$ & & & 6 \\
\hline Indo et al. [44] & $*$ & $*$ & $*$ & $*$ & $*$ & $*$ & & & 6 \\
\hline Ide et al. [45] & * & $*$ & $*$ & $*$ & $* *$ & $*$ & & & 7 \\
\hline Ide et al. [46] & $*$ & $*$ & $*$ & $*$ & $*$ & $*$ & & & 6 \\
\hline Jacques et al. [43] & $*$ & $*$ & $*$ & $*$ & $*$ & $*$ & & & 6 \\
\hline
\end{tabular}

ROS, representativeness of the exposed cohort; SOC, selection of nonexposed cohort; $A O E$, ascertainment of exposure; DOI, demonstration that the outcome of interest was not present at start of the study; AOU, assessment of outcome; DOF, degree of follow-up was long enough for outcomes to occur; AFU, adequacy of follow-up of cohorts.

R0 Resection Rate

Twenty studies described direct comparisons of the R0 resection rate. Six articles reported the $\mathrm{R} 0$ resection rate compared between P-ESD and C-ESD, and the pooled analysis showed that $\mathrm{P}-\mathrm{ESD}$ had higher $\mathrm{R} 0$ resection rate than C-ESD $(\mathrm{RR}=1.06,95 \% \mathrm{CI}[1.01,1.10], p<0.05)$ with a low heterogeneity $\left(I^{2}=21 \%, p=0.28\right)$. Three studies compared H-ESD with C-ESD, and analysis showed that there was no significant difference in the $\mathrm{R} 0$ resection rate between H-ESD and C-ESD (RR $=0.99,95 \%$ CI $[0.89$, $1.10], p=0.84)$ with a very low heterogeneity $\left(I^{2}=0 \%\right.$, $p=0.45)$. Ten articles reported the $\mathrm{R} 0$ resection rate compared between T-ESD and C-ESD, and the pooled analysis showed that T-ESD was similar to C-ESD $(\mathrm{RR}=1.03$, $95 \%$ CI $[0.99,1.07], p=0.18)$ with a relatively low heterogeneity $\left(I^{2}=41 \%, p=0.09\right)$. One article reported the R0 resection rate compared between T-ESD and P-ESD, and the analysis showed that T-ESD was similar to P-ESD (RR 


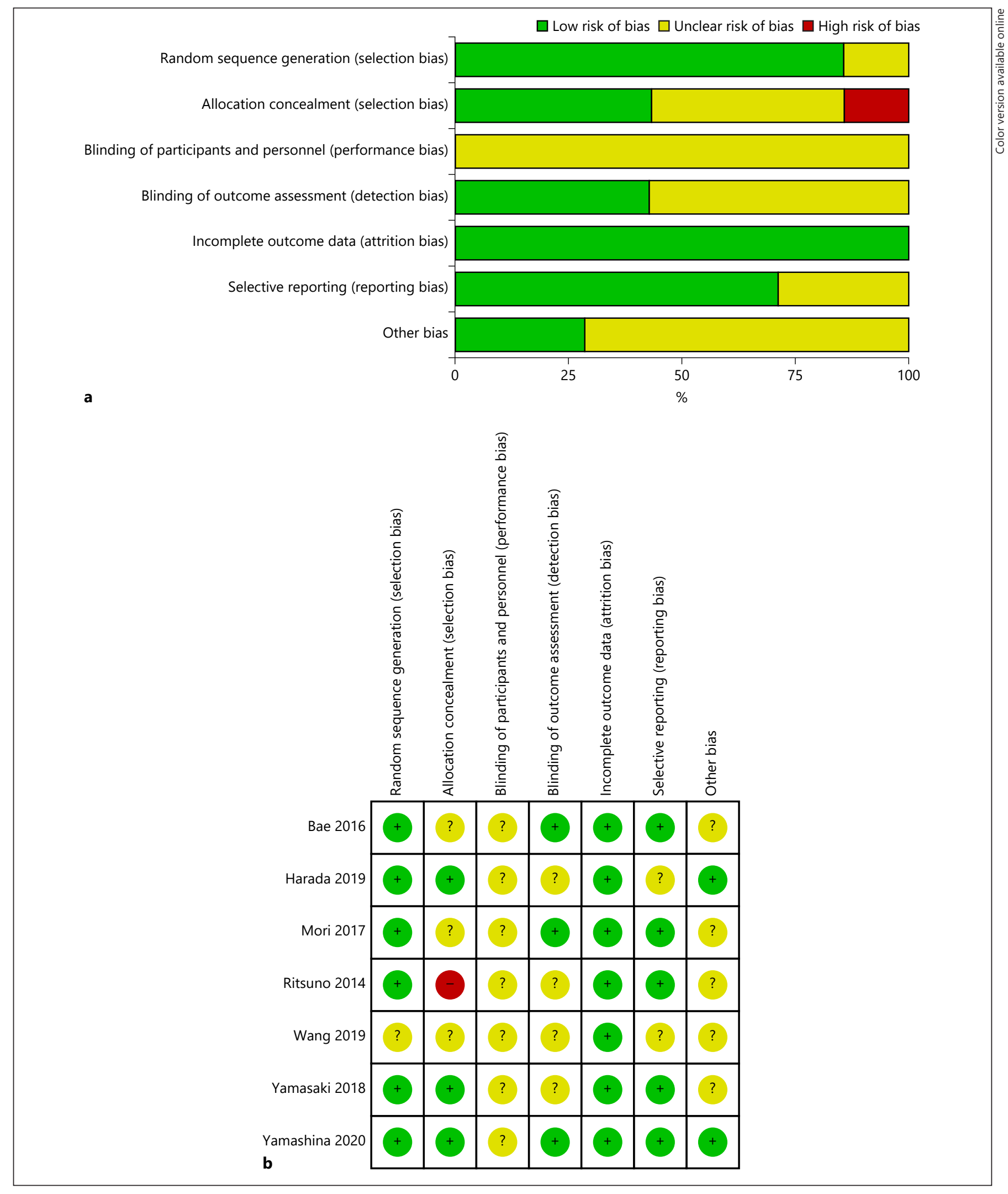

Fig. 2. a The risk of bias graph of RCT. b The risk of bias summary of RCT.

Effectiveness and Safety of Different Methods of Colorectal ESD
Dig Dis 2022;40:796-809

DOI: $10.1159 / 000521377$ 
$=1.22,95 \%$ CI $[1.00,1.47], p=0.05)$. The results are shown in online supplementary Figure 2.

\section{Operation Time}

Twenty-three studies described the comparison of operation time. Five articles reported the operation time compared between P-ESD and C-ESD, and the pooled analysis showed that P-ESD was significantly shorter than C-ESD (MD $=-7.86,95 \%$ CI $[-12.59,-3.13], p<$ $0.05)$ with a high heterogeneity $\left(I^{2}=54 \%, p=0.07\right)$. Jackknife sensitivity analysis indicated that the study by Yamashina et al. [24] was the primary source of heterogeneity, and this is likely because the study excluded patients who had more difficult surgery (online suppl. Fig. 7). Five studies compared H-ESD with C-ESD, and analysis showed the time of H-ESD was shorter than C-ESD (MD $=-21.29,95 \%$ CI $[-32.89,-9.69], p<0.05])$ with a high heterogeneity $\left(I^{2}=66 \%, p=0.02\right)$. Jackknife sensitivity analysis indicated that the study by Minalo et al. [40] was the primary source of heterogeneity, and this is likely because the endoscopists preferred H-ESD in the selection of surgery in this study (online suppl. Fig. 8). Twelve articles reported the operation time compared between TESD and C-ESD, and the pooled analysis showed that TESD was significantly shorter than C-ESD $(\mathrm{MD}=-23.83$, 95\% CI $[-34.28,-13.37], p<0.05)$ with a relatively high heterogeneity $\left(I^{2}=64 \%, p=0.001\right)$. Jackknife sensitivity analysis indicated that the studies by Indo et al. [44] and Yamashina et al. [33] were the primary sources of heterogeneity. The reasons may be that most patients were located in the deep colon, and endoscopists were not sufficiently skilled in T-ESD, respectively (online suppl. Fig. 9). One article reported the operation time compared between T-ESD and P-ESD, and the analysis showed that it was shorter for T-ESD than P-ESD (MD $=-22.30,95 \%$ CI $[-40.52,-4.08], p<0.05)$. The results are shown in online supplementary Figure 3.

\section{Dissection Speed}

Fourteen studies described the comparison of dissection speed. Six articles reported the dissection speed compared between P-ESD and C-ESD, and the pooled analysis showed that P-ESD was significantly faster than C$\mathrm{ESD}(\mathrm{MD}=4.25,95 \% \mathrm{CI}[3.58,4.91], p<0.05)$ with a low heterogeneity $\left(I^{2}=23 \%, p=0.26\right)$. One study compared $\mathrm{H}$-ESD with C-ESD, and analysis showed the dissection speed of $\mathrm{H}$-ESD was similar to C-ESD (MD $=6.0,95 \%$ CI $[-1.90,13.90], p=0.14)$. Six articles compared T-ESD and $\mathrm{C}-\mathrm{ESD}$, and the pooled analysis showed the dissection speed of T-ESD was similar to C-ESD (MD $=1.69,95 \%$
CI $[-7.36,10.73], p=0.71)$ with a very high heterogeneity $\left(I^{2}=98 \%, p<0.001\right)$. Sensitivity analysis indicated that the studies by Yamishina et al. [33] and Ide et al. [46] were the major sources of heterogeneity, in which the intervention group included more difficult deep colon lesions (online suppl. Fig. 10). One article reported the dissection speed compared between T-ESD and P-ESD, and the analysis showed that T-ESD was faster than P-ESD (MD $=11.50,95 \%$ CI $[6.68,16.32], p<0.05)$. The results are shown in online supplementary Figure 4.

\section{Adverse Events Rate}

All studies described the comparison of the adverse events rate. Six articles reported the adverse events rate compared between P-ESD and C-ESD, and the pooled analysis showed that $\mathrm{P}-\mathrm{ESD}$ had lower adverse events rate than C-ESD $(\mathrm{RR}=0.49,95 \% \mathrm{CI}[0.31,0.78], p<0.05)$ with a very low heterogeneity $\left(I^{2}=0 \%, p=0.42\right)$. Five studies compared H-ESD with C-ESD, and analysis showed that there was no significant difference in the adverse events rate between $\mathrm{H}$-ESD and C-ESD $(\mathrm{RR}=1.17,95 \% \mathrm{CI}[0.65$, $2.08], p=0.60)$ with a relatively low heterogeneity $\left(I^{2}=\right.$ $45 \%, p=0.12)$. Fourteen articles reported the adverse events rate compared between T-ESD and C-ESD, and the pooled analysis showed that T-ESD was similar to CESD (RR $=0.81,95 \%$ CI $[0.51,1.29], p=0.38)$ without heterogeneity $\left(I^{2}=0 \%, p=0.79\right)$. One article reported the adverse events rate compared between T-ESD and PESD, and the analysis showed that T-ESD was lower than $\mathrm{P}$-ESD $(\mathrm{RR}=0.41,95 \% \mathrm{CI}[0.20,0.85], p<0.05)$. The results are shown in online supplementary Figure 5.

\section{Network Meta-Analysis}

Network Plot

We drew five network evidence plots of five outcome indicators, each of which included C-ESD, P-ESD, HESD, and T-ESD, among which the node weight represented the total number of people and the edge weight represented the standard error. The summarized network plots of the comparisons are shown in Figure 3.

\section{En Bloc Resection Rate}

A total of 23 studies reported en bloc resection rate in different ESD groups. Network meta-analysis showed that the en bloc resection rate of $\mathrm{H}$-ESD was significantly lower than that of C-ESD, P-ESD, and T-ESD $(\mathrm{RR}=0.28$, $95 \% \mathrm{CI}[0.12,0.65] ; \mathrm{RR}=0.11,95 \% \mathrm{CI}[0.03,0.44] ; \mathrm{RR}=$ 8.28, 95\% CI [2.50, 27.42]; Fig. 4a). However, there was no significant difference between C-ESD, P-ESD, and T$\mathrm{ESD}$ in the en bloc resection rate. According to the cumu- 


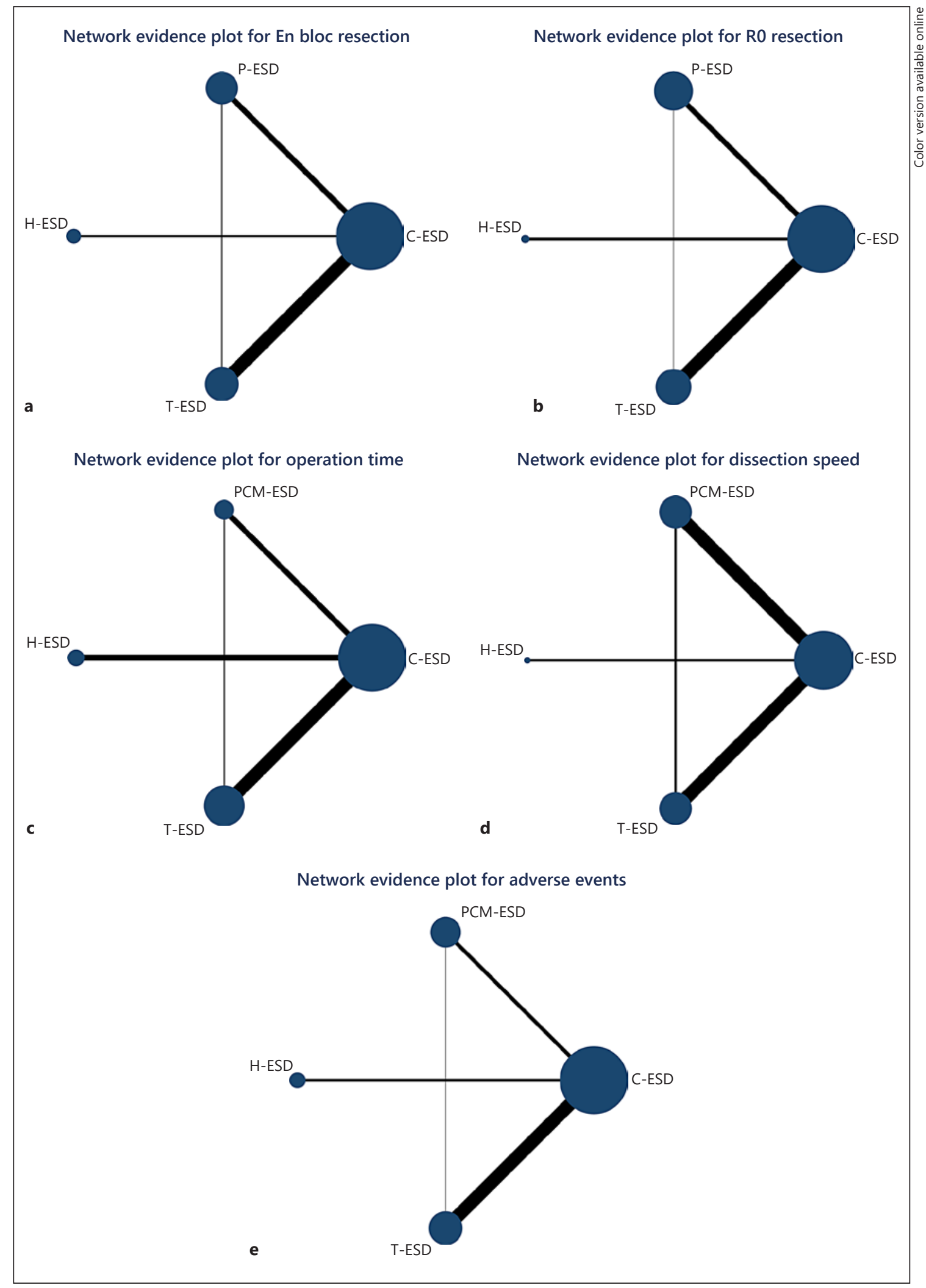

Fig. 3. a-e The network evidence plots of en bloc resection rate, R0 resection rate, operation time, dissection speed, and adverse events rate. 


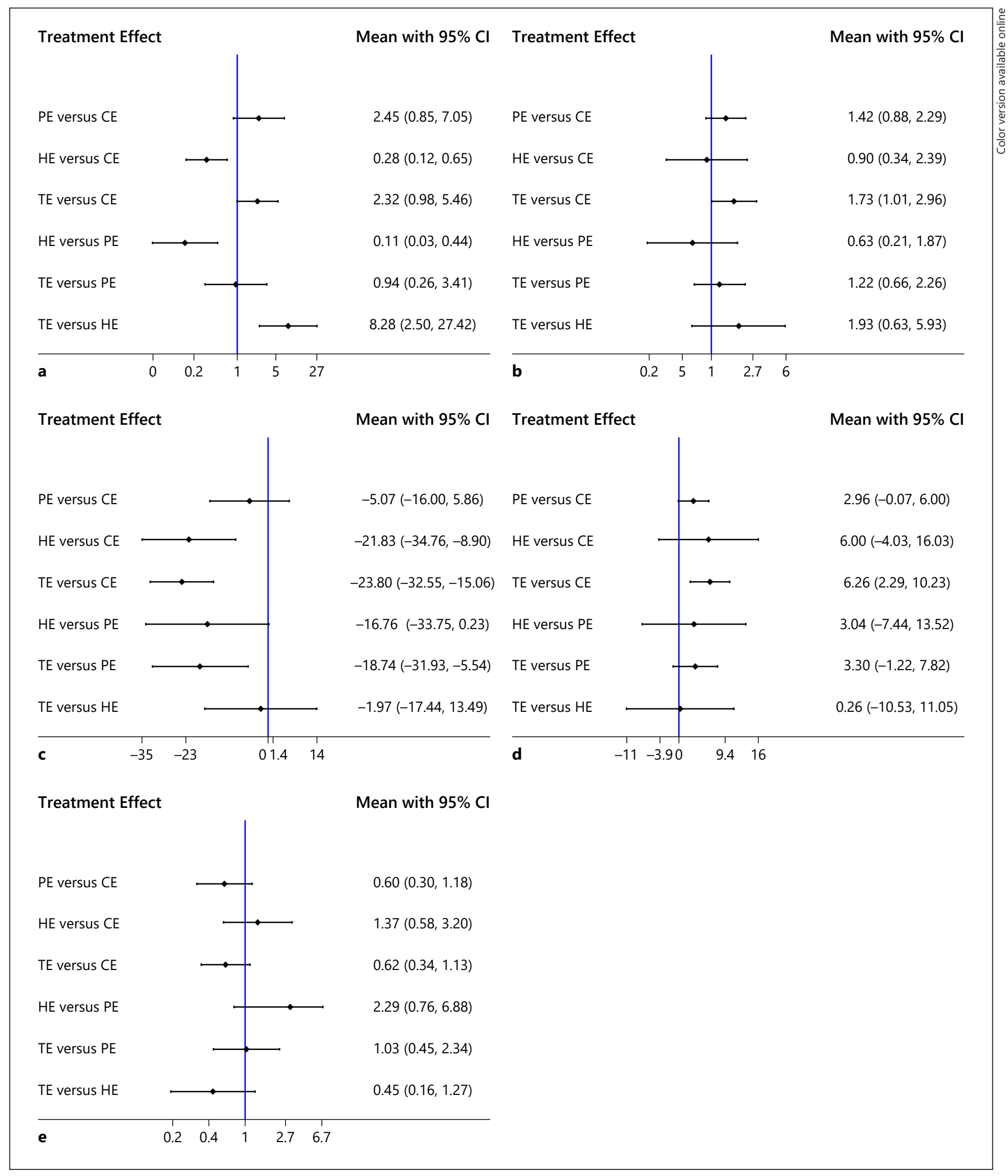

Fig. 4. a-e Forest plots represent the direct and indirect comparisons of en bloc resection rate, R0 resection rate, operation time, dissection speed, and adverse events rate. 


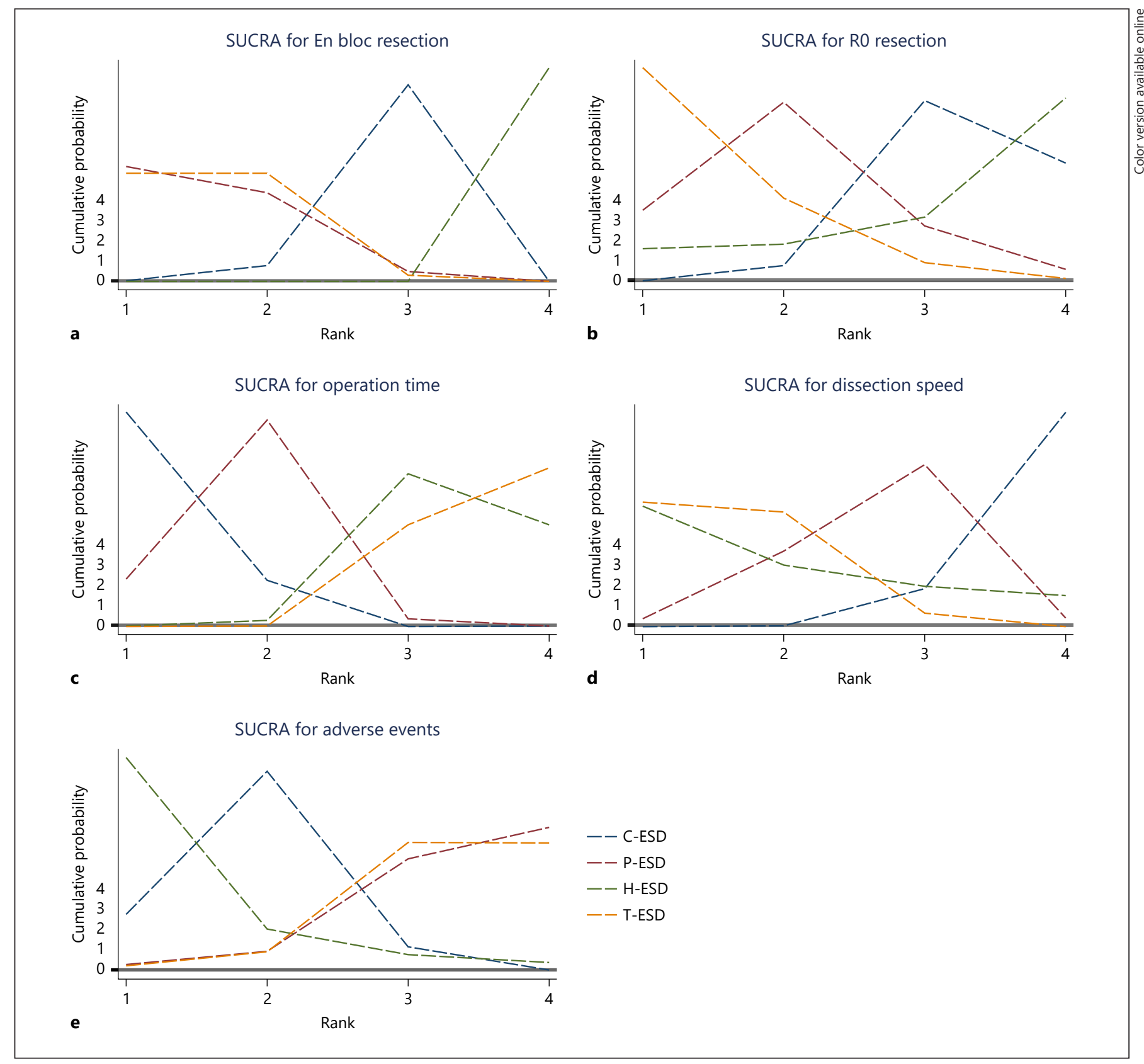

Fig. 5. a-e SUCRA for en bloc resection rate, R0 resection rate, operation time, dissection speed, and adverse events rate.

lative probability diagram, the en bloc resection rates of the four ESD methods were ranked as P-ESD, T-ESD, CESD, and H-ESD, respectively (Fig. 5a).

\section{R0 Resection Rate}

$\mathrm{R} 0$ resection rates in different $\mathrm{ESD}$ groups were compared in 20 qualified studies. The network meta-analy- sis revealed that T-ESD had a higher $\mathrm{R} 0$ resection rate than C-ESD ( $R R=1.73,95 \%$ CI [1.01, 2.96]; Fig. 4b). However, in other comparisons, no significant difference in the $\mathrm{R} 0$ resection rate was shown. We also ranked the $\mathrm{R} 0$ resection rates of the four ESD methods as TESD, P-ESD, C-ESD, and H-ESD, from high to low (Fig. 5b). 
Operation Time

Twenty-three studies compared operative times in different ESD groups. The NMAs results showed that compared with C-ESD, the time consumed by H-ESD and T-ESD was significantly shorter $(\mathrm{MD}=-21.83,95 \% \mathrm{CI}$ $[-34.76,-8.90]$; $\mathrm{MD}=-23.8,95 \%$ CI $[-32.55,-15.06]$; Fig. 4c). The operation time of T-ESD was also significantly shorter than that of P-ESD (MD $=-18.74,95 \%$ CI $[-31.93,-5.54]$; Fig. 4c). Meanwhile, we ranked the operation time of the four ESD methods as C-ESD, P-ESD, $\mathrm{H}-\mathrm{ESD}$, and T-ESD according to the cumulative probability diagram (Fig. 5c).

\section{Dissection Speed}

There were 11 quality studies that compared the dissection speed of different ESD groups. NMAs results exposed that the dissection speed of T-ESD was significantly faster than that of C-ESD (MD $=6.26,95 \%$ CI [2.29, 10.23]; Fig. 4d). There was no significant difference between the other comparison groups. According to the cumulative probability diagram, the dissection speed of the four ESD methods ranked as T-ESD, H-ESD, P-ESD, and C-ESD, from fast to slow (Fig. 5d).

\section{Adverse Events Rate}

All included studies compared the incidence of adverse events in different ESD groups. NMAs results showed that there was no significant difference in the adverse events rate among the four ESD procedures (Fig. 4e). Nevertheless, we ranked the slight differences between the four ESD methods according to the cumulative probability plot, with the adverse event rates from high to low being H-ESD, C-ESD, T-ESD, and P-ESD, respectively (Fig. 5e).

\section{Sensitivity Analysis}

We performed a separate analysis of the 7 included RCTs. The results of NMAs showed that there were no significant differences in en bloc resection rate, $\mathrm{R} 0$ resection rate, and adverse events rate among the four ESD procedures. Compared with C-ESD and P-ESD, the operation time of T-ESD was shorter $(\mathrm{MD}=-31.53,95 \% \mathrm{CI}$ $[-45.54,-17.52] ; \mathrm{MD}=-29.14,95 \% \mathrm{CI}[-50.77,-7.50])$. Compared with C-ESD, P-ESD and T-ESD dissected faster $(\mathrm{MD}=4.63,95 \% \mathrm{CI}[0.76,8.51] ; \mathrm{MD}=11.00,95 \% \mathrm{CI}$ [7.11, 14.89]). Meanwhile, T-ESD had a faster dissection speed than P-ESD (MD $=6.37,95 \%$ CI $[0.88,11.86])$. Moreover, the results of the predicted interval were consistent with the results of the appeal, suggesting a strong reliability of the NMA (online suppl. Fig. 11-12).
We also ranked different outcome measures for the four types of ESD according to cumulative probability plots. The en bloc resection rate from high to low was ranked as P-ESD, T-ESD, C-ESD, and H-ESD. The R0 resection rate was ranked from high to low as P-ESD, CESD, H-ESD, and T-ESD. The ranking of operation time from long to short was C-ESD, P-ESD, H-ESD, and TESD. The ranking of dissection speed from fast to slow was T-ESD, H-ESD, P-ESD, and C-ESD. And, the ranking of adverse events rate from most to least was H-ESD, C-ESD, P-ESD, and T-ESD (online suppl. Fig. 13).

\section{Publication Bias}

Sensitivity analysis was conducted for all studies with high heterogeneity, and potential causes were analyzed. We also assessed publication bias for all studies that included different outcome measures. The funnel plot for the adjustment of all results in NMAs showed that the corresponding studies were distributed roughly symmetric around the midline, indicating that there was no significant publication bias. Funnel plots for NMAs that included all studies and for NMAs that included only RCT studies are shown in online supplementary Figures 1415.

\section{Discussion}

Standard meta-analysis showed that P-ESD had superior en bloc resection rate, $\mathrm{R} 0$ resection rate, and dissection speed than C-ESD, with shorter operation time and lower incidence of adverse events. H-ESD mainly takes less time than C-ESD and speeds up the procedure, but otherwise makes no difference. Compared with C-ESD, the main advantages of T-ESD are that the dissection speed is significantly faster, the operation time is shorter, and the incidence of adverse events is not increased. However, T-ESD has little effect on the en bloc resection rate and $\mathrm{R} 0$ resection rate. In an individual study compared with P-ESD, T-ESD showed overall superiority. However, the comparison of H-ESD with P-ESD and TESD has not been reported. Although Jacques et al. [43] reported that T-ESD was superior to P-ESD in terms of en bloc resection, $\mathrm{R} 0$ resection, dissection speed, operative time, and adverse events, considering that this study was a retrospective study with a small sample size, the applicability of the results to the general population needs to be further verified. So, we further conducted a network meta-analysis to evaluate the advantages and disadvantages of the four ESD surgeries. 
The results of network meta-analysis showed that the main advantage of P-ESD was its higher en bloc resection rate, which ranked first among the four types of ESD surgeries. Meanwhile, P-ESD has shown certain advantages in surgical safety and ranked last among the four types of ESD in the incidence of adverse events. H-ESD mainly showed relative advantages in dissection speed and operation time, both ranked second. However, it had the worst performance in en bloc resection rate, $\mathrm{R} 0$ resection rate, and adverse events rate. T-ESD was the most prominent in dissection speed, and the operation time was significantly shortened, ranking the first among the four ESD operations. Moreover, T-ESD ranked highest in terms of en bloc resection rate, although the differences among the four ESD procedures were not significant.

P-ESD creates a submucosal pocket in which the tip of the endoscope is fixed, reducing the impact of pulse, respiration, and bowel movement on the operation, while reducing the risk of perforation and bleeding. In addition, studies have reported that saline-pocket ESD not only increased the traction force but also made the endoscopic field of vision clearer due to the refractive index of water [29]. Many previous studies have reported that P-ESD can significantly increase the speed of dissection, even in the cases of severe fibrosis or deep submucosal infiltration $[15,24]$. However, the results of our network metaanalysis did not show a significant advantage in the dissection speed of P-ESD, probably because some of our included studies excluded these patients with high difficulty of endoscopic procedures. Results from the NMAs, which included only RCT studies, showed a considerable advantage in dissection speed with P-ESD. T-ESD combines various traction devices on the basis of traditional ESD, such as floss, clip-line, clip-rubber band, S-O clip, medical ring, magnet, balloon, and robot platform [37, $41,43-45]$. Its main function is to provide better traction force for lesion dissection, accelerate the dissection speed, and shorten the operation process. Through the analysis of this study, both direct and indirect comparison, the dissection speed and operation time of T-ESD had the most obvious advantages among the four types of ESD. $\mathrm{H}-\mathrm{ESD}$ is a C-ESD with a snaring. After partial ESD dissection of the lesion, the remaining part is dissected by the snaring. Due to simplified surgical procedures, the operation time is shortened to a certain extent, which is consistent with the results of our NMAs. However, it has been reported in the previous literature that the risk of postoperative recurrence is gradually increased with the increase of lesion size, especially the lesion with a diameter greater than $20 \mathrm{~mm}$ [48]. This is mainly because large lesions of- ten require segmental resection. Our NMAs results showed that the en bloc resection rate of $\mathrm{H}-\mathrm{ESD}$ was significantly lower than that of the other three types of ESD. Compared with the results of pairwise meta-analysis, it was obvious that the results of our network meta-analysis were more reliable and more in line with the reality.

Due to the greater complexity, the effectiveness and safety of ESD depend to some extent on the skill of the endoscopist. According to the study by Gotoda et al. [49], a minimum of $40 \mathrm{ESD}$ procedures are required for the endoscopic trainee to overcome the learning curve and become proficient in the technique. When endoscopists who lack ESD experience complete the comparison between different ESD procedures, the conclusions will fluctuate and diverge to a large extent. Through sensitivity analysis, we found that the study of Indo et al. [44] was influenced by the learning curve, resulting in longer operation time in the T-ESD group than in the C-ESD group. However, the advantage of T-ESD was further demonstrated after the influence of the learning curve was excluded.

Since most of the literature studies included in this study were retrospective cohort studies, there may be some bias in the selection of patients and ESD methods, which leads to certain risks in the reliability of the results of this NMA. Therefore, we performed NMAs that included only RCT studies, and the results showed that the comparison of R0 resection rate, operation time, dissection speed, and incidence of adverse events was consistent with the previous analysis. This indicated that the robustness of the results of this NMA is good. The only significant inconsistency between the two network meta-analyses was the comparison of en bloc resection rates because the inclusion criteria for lesion size were different in all studies, and the en bloc resection rate of H-ESD was significantly lower for those patients with larger lesions.

Our study also had some limitations. First, many nonRCT studies were included in our study, and their methodological quality was somewhat deficient. Moreover, the sample included in some of the studies was small, so there might be small sample effect. Previous studies have also shown that the difficulty of operation is significantly increased when the lesions are located in the deep colon, which has a certain impact on the effectiveness and safety of ESD. Therefore, it is necessary to conduct subgroup analysis of different locations to further refine the advantages of different ESD methods.

Moreover, all ESD with traction devices were classified into the T-ESD group in this study, and the traction method was not further classified. Previous studies have shown that the effectiveness and safety of different T-ESD 
also have certain difference. This study mainly focused on the incidence of intraoperative and short-term postoperative adverse events, and because some studies did not even report the time range for the calculation of adverse events, the long-term prognosis of ESD was not further analyzed, which may be biased to some extent in the analysis of surgical safety.

\section{Conclusion}

P-ESD and T-ESD have the most outstanding comprehensive advantages in efficacy and safety among the four mainstream ESD methods for colorectal neoplasms. The main advantages of P-ESD are higher en bloc resection rate and less occurrence of adverse events. The main advantages of T-ESD are significantly faster dissection speed and shorter operation time. H-ESD has certain advantages in accelerating the dissection speed and shortening the operation time, but poor performance in the en bloc resection rate and $\mathrm{R} 0$ resection rate, so it is not considered as the first choice in the face of large lesions. This is of great clinical significance for endoscopists to choose different ESD procedures, but more high-quality RCT studies are needed for further verification in the future.

\section{Acknowledgments}

The authors are grateful to Dr. Jiyong Jing from Zhejiang Provincial People's Hospital, People's Hospital of Hangzhou Medical College, for constructive advice on statistical analysis. They also thank Dr. Wensheng Pan and Dr. Jiyong Jing for their assistance in manuscript translation and editing.

\section{Statement of Ethics}

An ethics statement is not applicable because this study is based exclusively on the published literature.

\section{Conflict of Interest Statement}

The authors declare that they have no conflicts of interest.

\section{Funding Sources}

This work was supported by the Zhejiang medicine key scientific and technology project (Grant No. 2018258924) and the Zhejiang medicine scientific and technology project (Grant No. 2019RC094) presided by Dr. Wensheng Pan and the Basic Public Welfare Research Project of Zhejiang Province (Grant No. LGF20F020018) presided by Dr. Jiyong Jing.

\section{Author Contributions}

(i) Conception and design: all authors; (ii) provision of study materials or patients: all authors; (iii) collection and assembly of data: Wu X.P., Ye X.L., Cao Z.S., and Jing J.Y.; (iv) data analysis and interpretation: Wu X.P., Ye X.L., Cao Z.S., Jing J.Y., and Pan W.S.; (v) manuscript writing: Wu X.P., Cao Z.S., and Hu X.C.; (vi) final approval of the manuscript: all authors.

\section{Data Availability Statement}

All data generated or analyzed during this study are included in this published article.

\section{References}

1 Sung H, Ferlay J, Siegel RL, Laversanne M, Soerjomataram I, Jemal A, et al. Global Cancer Statistics 2020: globocan estimates of incidence and mortality worldwide for 36 cancers in 185 countries. CA Cancer J Clin. 2021;71(3):209-49.

2 Shinozaki S, Hayashi Y, Lefor AK, Yamamoto $\mathrm{H}$. What is the best therapeutic strategy for colonoscopy of colorectal neoplasia? Future perspectives from the East. Dig Endosc. 2016;28(3): 289-95.

3 Saito Y, Uraoka T, Yamaguchi Y, Hotta K, Sakamoto $\mathrm{N}$, Ikematsu $\mathrm{H}$, et al. A prospective, multicenter study of 1,111 colorectal endoscopic submucosal dissections (with video). Gastrointest Endosc. 2010;72(6):1217-25.

4 De Ceglie A, Hassan C, Mangiavillano B, Matsuda T, Saito Y, Ridola L, et al. Endoscopic mucosal resection and endoscopic submucosal dis- section for colorectal lesions: a systematic review. Crit Rev Oncol Hematol. 2016;104 $138-55$.

5 Sakamoto N, Osada T, Shibuya T, Beppu K, Matsumoto K, Mori H, et al. Endoscopic submucosal dissection of large colorectal tumors by using a novel spring-action S-O clip for traction (with video). Gastrointest Endosc. 2009;69(7): 1370-4.

6 Saito Y, Emura F, Matsuda T, Uraoka T, Nakajima T, Ikematsu $\mathrm{H}$, et al. A new sinker-assisted endoscopic submucosal dissection for colorectal cancer. Gastrointest Endosc. 2005;62(2):297301.

7 Shi Q, Zhong PYS, Zhou PPH. Clinical value of dental floss tractionassisted endoscopic submucosa dissection for neuroendocrine neoplasm in rectum. J Gastroenterol Hepatol. 2019;34:80.
8 Abe S, Oda I, Suzuki H, Yoshinaga S, Saito Y. Insulated tip knife tunneling technique with clip line traction for safe endoscopic submucosal dissection of large circumferential esophageal cancer. VideoGIE. 2017;2(12):342-5.

9 Chinese Clinical Trial Registry. The safety and effectiveness of inner traction facilitated endoscopic submucosal resection using rubber band and clip in treating colorectal lateral spreading tumor: a randomized controlled trial. 2020. http: // www.who.int/trialsearch/Trial2. aspx?TrialID=ChiCTR2000039006.

10 Albouys J, Geyl S, Charissoux A, Legros R, Sautereau D, Pioche M, et al. Counter-traction using clips and rubber banding for endoscopic submucosal dissection of a laterally spreading tumor involving a diverticulum in the colon. Endoscopy. 2019;51(10):E295-6. 
11 Sakamoto N, Osada T, Ritsuno H, Murakami T, Shibuya T, Tomiki Y, et al. Endoscopic submucosal dissection of colorectal tumors using a traction device called the S-O clip. Gastroenterol Endosc. 2017;59(7):1514-23.

12 de Moura DT, Jirapinyo P, Farias GF, Hathorn K, Bazarbashi AN, Sachdev AH, et al. Robot-assisted ESD versus conventional ESD for colonic lesions: outcomes of a Randomized Pilot Study in endoscopists without prior ESD experience. Gastrointest Endoscopy. 2019;89(6):AB652-3.

13 Arezzo A, Forcignanò E, Morino M. Robotic endoscopic submucosal dissection and full-thickness excision for laterally spreading tumors of the rectum. Minim Invasive Ther Allied Technol. 2020:1-3.

14 Hayashi Y, Sunada K, Takahashi H, Shinhata H, Lefor AT, Tanaka A, et al. Pocket-creation method of endoscopic submucosal dissection to achieve en bloc resection of giant colorectal subpedunculated neoplastic lesions. Endoscopy. 2014;46 Suppl 1 UCTN:E421-2.

15 Sakamoto H, Hayashi Y, Miura Y, Shinozaki S, Takahashi H, Fukuda H, et al. Pocket-creation method facilitates endoscopic submucosal dissection of colorectal laterally spreading tumors, non-granular type. Endosc Int Open. 2017;5(2): E123-9.

16 Takezawa T, Hayashi Y, Shinozaki S, Sagara Y, Okada M, Kobayashi Y, et al. The pocket-creation method facilitates colonic endoscopic submucosal dissection (with video). Gastrointest Endosc. 2019;89(5):1045-53.

17 Kim YJ, Kim ES, Cho KB, Park KS, Jang BK, Chung WJ, et al. Comparison of clinical outcomes among different endoscopic resection methods for treating colorectal neoplasia. Dig Dis Sci. 2013;58(6):1727-36.

18 Toyonaga T, Man-I M, Morita Y, Azuma T. Endoscopic submucosal dissection (ESD) versus simplified/hybrid ESD. Gastrointest Endosc Clin N Am. 2014;24(2):191-9.

19 Liberati A, Altman DG, Tetzlaff J, Mulrow C, Gøtzsche PC, Ioannidis JP, et al. The PRISMA statement for reporting systematic reviews and meta-analyses of studies that evaluate healthcare interventions: explanation and elaboration. BMJ. 2009;339:b2700.

20 Higgins JP, Altman DG, Gøtzsche PC, Jüni P, Moher D, Oxman AD, et al. The Cochrane Collaboration's tool for assessing risk of bias in randomised trials. BMJ. 2011;343:d5928.

21 Wells G, Shea B, O'Connell J. The newcastle-ottawa Scale (NOS) for assessing the quality of nonrandomised studies in meta-analyses. Ottawa Health Research Institute; 2014. Web site 7.

22 Rücker G. Network meta-analysis, electrical networks and graph theory. Res Synth Methods. 2012;3(4):312-24.

23 Hozo SP, Djulbegovic B, Hozo I. Estimating the mean and variance from the median, range, and the size of a sample. BMC Med Res Methodol. 2005;5:13.

24 Yamashina T, Nemoto D, Hayashi Y, Fukuda H, Okada M, Takezawa T, et al. A prospective randomized trial comparing the pocket-creation method and conventional method of colorectal endoscopic submucosal dissection. Gastrointest Endosc. 2020;92(2):368-79.

25 Yamasaki Y, Takeuchi Y, Uedo N, Kanesaka T, Kato M, Hamada K, et al. Efficacy of traction-assisted colorectal endoscopic submucosal dissection using a clip-and-thread technique: a prospective randomized study (with videos). Gastroenterol Endosc. 2019;61(2): 192-204.

26 Wang F, Leng X, Gao Y, Zhao K, Sun Y, Bian H, et al. Endoscopic submucosal dissection of distal intestinal tumors using grasping forceps for traction. Tech Coloproctol. 2019;23(11):1079_ 83.

27 Ritsuno H, Sakamoto N, Osada T, Goto SP, Murakami T, Ueyama $\mathrm{H}$, et al. Prospective clinical trial of traction device-assisted endoscopic submucosal dissection of large superficial colorectal tumors using the S-O clip. Surg Endosc. 2014; 28(11):3143-9.

28 Mori H, Kobara H, Nishiyama N, Fujihara S, Matsunaga T, Masaki T. Novel effective and repeatedly available ring-thread counter traction for safer colorectal endoscopic submucosal dissection. Surg Endosc. 2017;31(7):3040-7.

29 Harada H, Nakahara R, Murakami D, Suehiro S, Ujihara T, Sagami R, et al. Saline-pocket endoscopic submucosal dissection for superficial colorectal neoplasms: a randomized controlled trial (with video). Gastrointest Endosc. 2019; 90(2):278-87.

30 Bae JH, Yang D-H, Lee S, Soh JS, Lee S, Lee H-S, et al. Optimized hybrid endoscopic submucosal dissection for colorectal tumors: a randomized controlled trial. Gastrointest Endosc. 2016; 83(3):584-92.

31 Terasaki M, Tanaka S, Oka S, Nakadoi K, Takata $\mathrm{S}$, Kanao H, et al. Clinical outcomes of endoscopic submucosal dissection and endoscopic mucosal resection for laterally spreading tumors larger than $20 \mathrm{~mm}$. J Gastroenterol Hepatol. 2012; 27(4):734-40.

32 Ye L, Yuan X, Pang M, Bethge J, Ellrichmann $\mathrm{M}, \mathrm{Du}$ J, et al. Magnetic bead-assisted endoscopic submucosal dissection: a gravity-based traction method for treating large superficial colorectal tumors. Surg Endosc. 2019;33(6): 2034-41.

33 Yamashina T, Hayashi Y, Sakamoto H, Yano T, Miura Y, Shinozaki S, et al. Balloon-assisted endoscopy facilitates endoscopic submucosal dissection of difficult superficial proximal colon tumors. Endoscopy. 2018;50(8):800-8.

34 Yamashina T, Hayashi Y, Fukuda H, Okada M, Takezawa T, Kobayashi Y, et al. The pocket-creation method may facilitate endoscopic submucosal dissection of large colorectal sessile tumors. Endosc Int Open. 2020;8(8):E1021-30.

35 Yamada S, Doyama H, Ota R, Takeda Y, Tsuji K, Tsuij S, et al. Impact of the clip and snare method using the prelooping technique for colorectal endoscopic submucosal dissection. Endoscopy. 2016;48(3):281-5

36 Takashiro H, Saito H, Tawada K, Oyama Y, Tazawa S, Usui M, et al. Efficacy of early clipwith-line method for colorectal endoscopic submucosal dissection. Surg Endosc. 2021.
37 Okamoto Y, Oka S, Tanaka S, Inagaki K, Tanaka $\mathrm{H}$, Matsumoto K, et al. Clinical usefulness of the S-O clip during colorectal endoscopic submucosal dissection in difficult-to-access submucosal layer. Endosc Int Open. 2020;8(3):E437-44.

38 Okamoto K, Muguruma N, Kitamura S, Kimura T, Takayama T. Endoscopic submucosal dissection for large colorectal tumors using a crosscounter technique and a novel large-diameter balloon overtube. Dig Endosc. 2012;24(Suppl 1): 96-9.

39 Okamoto K, Muguruma N, Kagemoto K, Mitsui Y, Fujimoto D, Kitamura S, et al. Efficacy of hybrid endoscopic submucosal dissection (ESD) as a rescue treatment in difficult colorectal ESD cases. Dig Endosc. 2017;29 Suppl 2:45-52.

40 Milano RV, Viale E, Bartel MJ, Notaristefano C, Testoni PA. Resection outcomes and recurrence rates of endoscopic submucosal dissection (ESD) and hybrid ESD for colorectal tumors in a single Italian center. Surg Endosc. 2018;32(5): 2328-39.

41 Kuroki Y, Asonuma K, Uehara N, Endo T, Suzuki R, Yamamoto Y, et al. Efficacy and suitable indication of colorectal endoscopic submucosal dissection using a balloon-assisted endoscope. JGH Open. 2020;4(2):185-90.

42 Kanamori A, Nakano M, Kondo M, Tanaka T, Abe K, Suzuki T, et al. Clinical effectiveness of the pocket-creation method for colorectal endoscopic submucosal dissection. Endosc Int Open. 2017;5(12):E1299-305.

43 Jacques J, Charissoux A, Bordillon P, Legros R, Rivory J, Hervieu V, et al. High proficiency of colonic endoscopic submucosal dissection in Europe thanks to countertraction strategy using a double clip and rubber band. Endosc Int Open. 2019;7(9):E1166-74.

44 Indo N, Anami T, Kitamura Y, Watanabe A. Effectiveness of counter traction using a clip with a looped thread for colorectal endoscopic submucosal dissection. Endosc Int Open. 2021;9(3): E306-12.

45 Ide D, Saito S, Ohya TR, Nishikawa Y, Horie Y, Yasue C, et al. Colorectal endoscopic submucosal dissection can be efficiently performed by a trainee with use of a simple traction device and expert supervision. Endosc Int Open. 2019;7(6): E824-32.

46 Ide D, Ohya TR, Saito S, Mitsuyoshi Y, Hatamori $\mathrm{H}$, Ikenoyama $\mathrm{Y}$, et al. Clinical utility of the pocket-creation method with a traction device for colorectal endoscopic submucosal dissection. Surg Endosc. 2021;35(5):2110-8.

47 Byeon JS, Yang DH, Kim KJ, Ye BD, Myung SJ, Yang SK, et al. Endoscopic submucosal dissection with or without snaring for colorectal neoplasms. Gastrointest Endosc. 2011;74(5):107583.

48 Tajika M, Niwa Y, Bhatia V, Kondo S, Tanaka T, Mizuno N, et al. Comparison of endoscopic submucosal dissection and endoscopic mucosal resection for large colorectal tumors. Eur J Gastroenterol Hepatol. 2011;23(11):1042-9.

49 Gotoda T, Friedland S, Hamanaka H, Soetikno R. A learning curve for advanced endoscopic resection. Gastrointest Endosc. 2005;62(6):866-7.
Effectiveness and Safety of Different Methods of Colorectal ESD
Dig Dis 2022;40:796-809

DOI: $10.1159 / 000521377$ 